\title{
Orlicz equi-integrability for scaled gradients
}

\author{
Piotr Antoni Kozarzewski ${ }^{1,2} \cdot$ Elvira Zappale ${ }^{3}$
}

Abstract In the realm of $3 D-2 D$ dimensional reduction problems, we prove that, up to an extraction, it is possible to decompose a sequence $\left(u_{n}\right)$, whose scaled gradients $\left(\nabla_{\alpha} u_{n}, \frac{1}{\varepsilon_{n}} \nabla_{3} u_{n}\right)$ are bounded in $L^{\Phi}\left(\omega \times(-1,1), \mathbb{R}^{3 \times 3}\right)$ for a suitable Orlicz function $\Phi$, as $u_{n}=v_{n}+z_{n}$, such that $v_{n}$ describes the oscillations, $\left(\Phi\left(\left|\nabla_{\alpha} v_{n}, \frac{1}{\varepsilon_{n}} \nabla_{3} v_{n}\right|\right)\right)$, is equi-integrable and the remainder $z_{n}$, accounting for concentration effects, converges to zero in measure. In particular, we extend to the Orlicz-Sobolev setting the results contained in Bocea and Fonseca, (ESAIM: COCV 7:443-470, 2002) and Braides and Zeppieri (Calc Var 29:231-238, 2007).

Keywords Equi-integrability $\cdot$ Scaled gradients · Orlicz-Sobolev spaces

Mathematics Subject Classification Primary 74B20 - 74K35 - 74K15 .

Secondary 49J45

Piotr Antoni Kozarzewski

piotr.kozarzewski@mimuw.edu.pl

Elvira Zappale

ezappale@unisa.it

1 Faculty of Mathematics, Informatics and Mechanics, University of Warsaw, ul. Banacha 2, 02-097 Warsaw, Poland

2 Faculty of Cybernetics, Military University of Technology, ul. gen. Sylwestra Kaliskiego 2, 00-908 Warsaw, Poland

3 Department of industrial Engineering, Universitá di Salerno, via Giovanni Paolo II, 132, 84084 Fisciano, SA, Italy 


\section{Introduction}

In the study of thin structures, i.e. when one or more dimensions are much smaller than the others, say of order $\varepsilon<<1$, rigorous analysis via dimensional reduction proves to be a useful tool to deduce properties of thin domains starting from thicker models. In this analysis one deals with sequences of functions defined on cylindrical sets with some thin ( $\varepsilon$ sized) dimension. In the $3 D$ setting, thin films are modelled as $\omega \times(-\varepsilon, \varepsilon)$ with $\omega \subset \mathbb{R}^{2}$ a bounded open set. In order to perform an asymptotic analysis as $\varepsilon \rightarrow 0$, with the aim of deducing a theory settled in $\omega$, functions are usually rescaled to an $\varepsilon$-independent reference configuration, so that a new sequence $\left(u_{\varepsilon}\right)$ is constructed, satisfying, in the standard Sobolev setting, some 'degenerate' bounds of the form

$$
\int_{\omega \times(-1,1)}\left(\left|\nabla_{\alpha} u_{\varepsilon}\right|^{p}+\frac{1}{\varepsilon^{p}}\left|\nabla_{3} u_{\varepsilon}\right|^{p}\right) d x \leq C<+\infty,
$$

if the sequence of unscaled gradients $\left(\nabla w_{\varepsilon}\right)$ satisfied some corresponding $L^{p}$ bound on the unscaled domain $\omega \times(-\varepsilon, \varepsilon)$.

Above and in the sequel $\nabla_{\alpha}$ represents the gradient with respect to the unscaled coordinates (denoted by $x_{\alpha}$ ) and $\nabla_{3}$ represents the gradient with respect to the thin coordinate direction denoted by $x_{3}$. In particular, $\Omega:=\omega \times(-1,1)=\left\{\left(x_{\alpha}, x_{3}\right)\right.$ : $\left.\left(x_{\alpha}, \varepsilon x_{3}\right) \in \omega \times(-\varepsilon, \varepsilon)\right\}$ and $u_{\varepsilon}\left(x_{\alpha}, x_{3}\right)=w_{\varepsilon}\left(x_{\alpha}, \varepsilon x_{3}\right)$.

Bocea and Fonseca in [3] (see also Braides and Zeppieri in [4] for any dimension) proved an equi-integrability Lemma for scaled gradients satisfying a bound as (1). Indeed they generalized the Fonseca et al.'s result (see [7, Lemma 1.2], in turn refining the results in [1]) which allows to substitute a sequence $\left(u_{n}\right)$, whose gradients $\left(\nabla u_{n}\right)$ are bounded in $L^{p}$, by a sequence $\left(v_{n}\right)$ with $\left(\left|\nabla v_{n}\right|^{p}\right)$ equiintegrable, such that the two sequences are equal except on a set of vanishing measure. The purpose of such a result is due to the fact that when applying the direct methods of the Calculus of Variations, or some $\Gamma$-convergence argument, it is very convenient to replace a given sequence with one having better regularity and integrability properties.

In this note we extend [3, Theorem 1.1, Corollary 1.2] to the Orlicz-Sobolev setting (see Sect. 2 for details and properties about Orlicz spaces $L^{\Phi}$ and OrliczSobolev ones $W^{1, \Phi}$ ). Our main motivation is to provide new tools, namely the Lipschitz type approximation for scaled gradients, to the asymptotic analysis of thin structures whose stored energy can be modelled in terms of Orlicz-Sobolev functions. Indeed a larger class of materials can be considered, replacing standard coercivity and growth condtions (i.e. of the type $|\cdot|^{p}$ ) for the energy density, by convex functions [satisfying suitable properties, as (5) and (6)]. We refer to the recent works [18, 19] aimed to describe thin structures and their bending phenomena, and to the forthcoming paper [16], where optimal design questions are addressed in the same spirit of $[5,6]$. We believe that our result can have further applications like those to fluid mechanics and multiscale problems (we refer to [21], where homogenization of integral functionals was treated, in a very similar setting to ours). 
Via Young measures techniques, we prove

Theorem 1 Let $\omega \subset \mathbb{R}^{2}$ be a bounded open set with Lipschitz boundary and $\Omega:=\omega \times(-1,1)$. Let $\Phi:[0,+\infty) \rightarrow[0,+\infty)$ be an Orlicz function satisfying (5) and (6). Let $\left(u_{n}\right) \subset W^{1, \Phi}\left(\Omega ; \mathbb{R}^{3}\right)$. Assume that $\left(\varepsilon_{n}\right)$ is a sequence of numbers converging to 0 , such that

$$
\sup _{n} \int_{\Omega}\left(\Phi\left(\left|\nabla_{\alpha} u_{n}, \frac{1}{\varepsilon_{n}} \nabla_{3} u_{n}\right|\right)\right) d x=C<+\infty .
$$

Then there exists a (non-relabelled) subsequence $\left(u_{n}\right)$ and a sequence $\left(v_{n}\right) \subset$ $W^{1, \Phi}\left(\Omega ; \mathbb{R}^{3}\right)$ such that

(i) sequence $\left(\Phi\left(\left|\nabla_{\alpha} v_{n}, \frac{1}{\varepsilon_{n}} \nabla_{3} v_{n}\right|\right)\right)$ is equi-integrable,

(ii) $\quad v_{n} \rightarrow u_{0}$ in $W^{1, \Phi}\left(\Omega ; \mathbb{R}^{3}\right)$, where $u_{0}$ is the weak limit of $\left(u_{n}\right)$ in $W^{1, \Phi}\left(\Omega ; \mathbb{R}^{3}\right)$,

(iii) $\mid\left\{x \in \Omega: u_{n} \neq v_{n}\right.$ or $\left.\nabla u_{n} \neq \nabla v_{n}\right\} \mid \rightarrow 0$, as $n \rightarrow+\infty$,

(iv) $\quad v_{n \mid \partial \omega \times(-1,1)}=u_{0}$.

We stress that the above result holds for any sequence of scaled gradients appearing in any $N d-K d$ dimensional reduction problem, besides the proof is presented for $N=3$ and $K=2$.

Having in mind the equilibrium problems related to membranes, where the total energy of the thin film under a deformation $w_{\varepsilon}: \omega \times(-\varepsilon, \varepsilon) \rightarrow \mathbb{R}^{3}$ is given by

$$
E_{\varepsilon}\left(w_{\varepsilon}\right):=\int_{\omega \times(-\varepsilon, \varepsilon)} W\left(\nabla w_{\varepsilon}(y)\right) d y-\int_{\omega \times(-\varepsilon, \varepsilon)} f^{\varepsilon}(y) \cdot w_{\varepsilon}(y) d y,
$$

with $f^{\varepsilon} \in L^{\Psi}\left(\omega \times(-\varepsilon, \varepsilon), \mathbb{R}^{3}\right)$ an appropriate dead loading body force density (we refer to [18] for the asymptotic analysis of the above energy), it is important to prove the existence of an 'attaining' sequence for the limit density, which is $\Phi$-equiintegrable. Indeed the following result holds.

Theorem 2 Let $\Omega$ and $\Phi$ be as in Theorem 1 . Let $u_{0} \in W^{1, \Phi}\left(\omega, \mathbb{R}^{3}\right)$ be an affine mapping with gradient $\xi_{0} \in \mathbb{R}^{3 \times 2}$ and let $W: \mathbb{R}^{3 \times 3} \rightarrow \mathbb{R}$ be a continuous function satisfying

$$
\beta \Phi(|\xi|)-c \leq W(\xi) \leq \beta^{\prime} \Phi(|\xi|)+C \text { forevery } \xi \in \mathbb{R}^{3 \times 3},
$$

for suitable constant $0<\beta \leq \beta^{\prime}, c, C>0$.

Given any sequence $\left(\varepsilon_{n}\right)$ of positive real numbers converging to zero, there exist a subsequence (not relabelled) of $\left(\varepsilon_{n}\right)$, and a sequence of functions $\left(u_{n}\right) \subset$ $W^{1, \Phi}\left(\Omega, \mathbb{R}^{3}\right)$ such that

(i) $\lim _{n \rightarrow+\infty} \frac{1}{|\Omega|} \int_{\Omega} W\left(\nabla_{\alpha} u_{n}, \frac{1}{\varepsilon_{n}} \nabla_{3} u_{n}\right) d x=Q \bar{W}\left(\xi_{0}\right)$, where $\bar{W}\left(\xi_{0}\right)=\min _{z \in \mathbb{R}^{3}}$ $W\left(\xi_{0} \mid z\right)$ and $Q \bar{W}$ denotes the quasiconvex envelope of $\bar{W}$, namely 


$$
Q \bar{W}\left(\xi_{0}\right)=\inf _{\varphi \in W_{0}^{1, \infty}\left(Q_{b}, \mathbb{R}^{3}\right)}\left\{\left|Q_{b}\right|^{-1} \int_{Q_{b}} \bar{W}\left(\xi_{0}+\nabla_{\alpha} \varphi\left(x_{\alpha}\right)\right) d x_{\alpha}\right\}
$$

for any cube $Q_{b} \subseteq \omega$,

(ii)

(iii) $\quad u_{n \mid \partial \omega \times(-1,1)}=u_{0}$.

(iv) $\Phi\left(\left|\nabla_{\alpha} u_{n}, \frac{1}{\varepsilon_{n}} \nabla_{3} u_{n}\right|\right)$ is equi-integrable.

It is worth to observe that such a result can be seen as a counterpart of the characterization of the Young measures generated by scaled gradients in the OrliczSobolev setting. Indeed formula (i) is entirely analogous to [14, formula before (1.16)].

The proof of Theorem 1 develops first by proving a Decomposition Lemma for standard gradients (see Theorem 4) which relies on properties of maximal functions, and exploits the Fundamental Theorem of Young measures (see Theorem 3). Then the proof of Theorem 1 follows as a consequence making use of the fine homogenization technique introduced in [4]. These are the subject of Sect. 3, while all the preliminary results, together with properties of Hardy maximal operator are contained in Sect. 2.

\section{Notation and preliminaries}

We will use the following notation:

- $|A|$ denotes the Lebesgue measure of a set $A$ in $\mathbb{R}^{N}, N \geq 2$, and it will be clear from the context;

- the symbol $d x$ will also be used to denote integration with respect to the Lebesgue measure $\mathcal{L}^{N}, N \geq 3$;

- the symbol $d x_{\alpha}$ will be used to denote integration with respect to the Lebesgue measure $\mathcal{L}^{2}$;

- the symbol $\nabla_{\alpha} u$ denotes the derivatives with respect to $x_{\alpha}:=\left(x_{1}, x_{2}\right)$ of a given field $u$;

- $C$ represents a generic positive constant that may change from line to line;

- a matrix $\xi \in \mathbb{R}^{3 \times 3}$, will be often written as $\left(\xi_{\alpha}, \xi_{3}\right)$ where $\xi_{\alpha}$ stands for the first two columns and $\xi_{3}$ represents the third;

- the Euclidean norm of a vector or of a matrix will be described as $|\cdot|$ and it will be clear from the context;

- a sequence $\left(f_{n}\right)$ is said to be $\Phi$-equi-integrable if the sequence $\left(\Phi\left(\left|f_{n}\right|\right)\right)$ is equiintegrable.

We say that $\Phi:[0,+\infty) \rightarrow[0,+\infty)$ is an Orlicz function whenever it is continuous, strictly increasing, convex, vanishes only at 0 and $\lim _{t \rightarrow 0^{+}} \Phi(t) / t=$ $0 ; \lim _{t \rightarrow+\infty} \Phi(t) / t=+\infty$. This statement is equivalent to demanding that $\Phi(t)=$ 
$\int_{0}^{t} \phi(s) d s$ for some right-continuous, non-decreasing $\phi$ s.t. $\phi(t)=0 \Longleftrightarrow t=0$ and $\lim _{t \rightarrow+\infty} \phi(t)=+\infty$.

We say that $\Phi$ satisfies $\Delta_{2}$ (denoted by $\Phi \in \Delta_{2}$ ) condition whenever

$$
\text { there exist } C>0 \text { and } t \geq t_{0} \text { such that } \Phi(2 t)<C \Phi(t) \text { for all } t \geq t_{0} \text {. }
$$

Orlicz functions $\Phi$ possess the complementary Orlicz function $\Psi(s):=\Phi^{\star}(s)$, where the latter denotes the standard Fenchel's conjugate of $\Phi$, i.e.

$$
\Psi(s):=\sup _{t \geq 0}\{s t-\Phi(t)\}, \quad s \geq 0,
$$

and, it results that $\Psi(s)=\int_{0}^{s} \phi^{-1}(\tau) d \tau$, where $\phi^{-1}$ stands for right inverse function of $\phi$.

Clearly $\Psi^{\star}=\left(\Phi^{\star}\right)^{\star}=\Phi$.

If $\Psi \in \Delta_{2}$ then (see [17, Theorem 4.2])

there exist $C>0$ and $t_{0} \geq 0$ such that $\Phi(t) \leq 1 /(2 C) \Phi(C t)$ for any $t>t_{0}$.

Given two Orlicz functions $\Phi$ and $\Phi^{\prime}, \Phi$ dominated $\Phi^{\prime}$ near infinity $\left(\Phi^{\prime} \prec \Phi\right.$ or $\Phi \succ \Phi^{\prime}$ in symbols) if there exists $C>1$ and $t_{0}>0$ such that $\Phi^{\prime}(t) \leq \Phi(C t)$ for all $t>t_{0}$.

For an arbitrary set of positive Lebesgue measure $E \subset \mathbb{R}^{N}$ we define the Orlicz class $L_{\Phi}(E)$ of functions $u$ on $E$ as functions satisfying inequality

$$
\int_{E} \Phi(|u|) d x<+\infty
$$

In general the class $L_{\Phi}(E)$ is not a linear space, and the Orlicz space $L^{\Phi}(E)$ is defined as the linear hull of $L_{\Phi}(E)$. It is easy to check that (see [17, Theorem 8.2]) Orlicz class $L_{\Phi}(E)$ coincides with its Orlicz space $L^{\Phi}(E)$ if and only if $\Phi \in \Delta_{2}$.

Orlicz spaces are equipped with the Luxemburg norm, namely

$$
\|u\|_{L^{\Phi}(E)}=\inf _{k>0} \int_{E} \Phi(|u| / k) \leq 1
$$

and are complete (see [17, Theorems 9.2 and 9.5]).

The following properties hold.

Lemma 1 Let $\Phi$ be an Orlicz function satisfying the $\Delta_{2}$ condition (i.e. (5)) and let E be a bounded open set in $\mathbb{R}^{N}$. Then

(i) $C_{c}^{\infty}(E)$ is dense in $L^{\Phi}(E)[10$, Theorem 1];

(ii) $\quad L^{\Phi}(E)$ is separable [17, point 4 at page 85$]$ and it is reflexive when $\Phi$ satisfies (6) [17, Theorem 14.2];

(iii) the dual of $L^{\Phi}(E)$ is identified with $L^{\Psi}(E),\left(\Psi=\Phi^{\star}\right)$ and the dual norm on $L^{\Psi}(E)$ is equivalent to $\|\cdot\|_{L^{\Psi}}[17$, Theorem 14.2]; 
(iv) given $u \in L^{\Phi}(E)$ and $v \in L^{\Psi}(E)$, then $u v \in L^{1}(E)$ and the following generalized Hölder inequality holds [17, Theorem 9.3 and formula (9.24)]

$$
\left|\int_{E} u v d x\right| \leq 4\|u\|_{L^{\phi}}\|v\|_{L^{\Psi}}
$$

(v) for every $v \in L^{\Phi}(E)$ the linear functional $I_{v}$ on $L^{\Psi}(E)$ defined as

$$
I_{v}(u):=\int_{E} u(x) v(x) d x
$$

belongs to the dual of $L^{\Psi}(E)$ with $\|v\|_{L^{\Phi}} \leq\left\|L_{v}\right\|_{\left[L^{\Psi}(E)\right]^{\prime}} \leq 2\|v\|_{L^{\Phi}}[17$, Theorem 9.5, formula 9.24];

(vi) given $\Phi$ and $\tilde{\Phi}$, the continuous embedding $L^{\Phi}(E) \hookrightarrow L^{\tilde{\Phi}}(E)$ holds iff $\Phi \succ \tilde{\Phi}[17$, Theorem 8.1];

(vii) $\quad$ in view of (vi) $L^{\Phi}(E) \hookrightarrow L^{1}(E) \hookrightarrow L_{\mathrm{loc}}^{1}(E) \hookrightarrow \mathcal{D}^{\prime}(E)$;

(viii) the product of d identical copies of $L^{\Phi}(E),\left(L^{\Phi}(E)\right)^{d}:=L^{\Phi}(E) \times \ldots \times$ $L^{\Phi}(E)$ endowed with the norm $\|v\|_{\left(L^{\Phi}(E)\right)^{d}}:=\sum_{i=1}^{d}\left\|v_{i}\right\|_{L^{\Phi}(E)}$ is an Orlicz space (i.e. the norm is equivalent to the $L^{\Phi}\left(\sqcup_{1}^{d} E\right)$ norm, where $\sqcup$ stays for sum of disjoint copies of the set).

Sobolev-Orlicz spaces $W^{1, \Phi}(E)$ are defined as follows

$$
W^{1, \Phi}(E):=\left\{u \in \mathcal{D}^{\prime}(E): u \in L^{\Phi}(E), \nabla u \in\left(L^{\Phi}(E)\right)^{N}\right\}
$$

endowed with the norm

$$
\|u\|_{W^{1, \Phi}(E)}:=\|u\|_{L^{\Phi}(E)}+\|\nabla u\|_{\left(L^{\Phi}(E)\right)^{N}}
$$

thus they are Banach spaces.

The Sobolev-Orlicz space $W^{1, \Phi}\left(E ; \mathbb{R}^{d}\right), d \in \mathbb{N}$ is defined as the Banach space of $\mathbb{R}^{d} \quad$ valued functions $u \in L^{\Phi}\left(E ; \mathbb{R}^{d}\right)$ with distributional derivative $\nabla u \in L^{\Phi}\left(E ; \mathbb{R}^{N \times d}\right)$, equipped with the norm

$$
\|u\|_{W^{1, \Phi}\left(E ; \mathbb{R}^{d}\right)}:=\|u\|_{L^{\Phi}\left(E ; \mathbb{R}^{d}\right)}+\|\nabla u\|_{L^{\Phi}\left(E ; \mathbb{R}^{N \times d}\right)},
$$

where the meaning of the norm $\|\cdot\|_{L^{\Phi}\left(E ; \mathbb{R}^{\cdot}\right)}$ is easily understood from (viii) in Lemma 1. On the other hand, all the other properties in Lemma 1 extend with obvious meaning to the vectorial setting.

If $E$ has Lipschitz boundary, then the embedding

$$
W^{1, \Phi}\left(E ; \mathbb{R}^{d}\right) \hookrightarrow L^{\Phi}\left(E ; \mathbb{R}^{d}\right)
$$

is compact (see [2] and [9, Theorems 2.2 and Proposition 2.1]).

For Sobolev-Orlicz space $W^{1, \Phi}(E)$, where $E$ has a Lipschitz boundary and $\Phi \in \Delta_{2}$, there exists a linear continuous trace operator $\operatorname{Tr}: W^{1, \Phi}(E) \rightarrow L^{\Phi}(\partial E)[11$, Theorem 3.13]. 
Let $\mathcal{M}$ be a (centred) Hardy maximal operator, i.e. for any $f \in L_{l o c}^{1}(E) \cap L^{\Phi}(E)$ let

$$
\mathcal{M} f(x):=\sup _{r}|B(x, r)|^{-1} \int_{B(x, r) \cap E}|f(x)| d x .
$$

The following result will be exploited in the sequel.

Proposition 1 (Weak estimate on Hardy's operator) Let $\Phi$ be an Orlicz function satisfying (5) and (6). For any $f \in L^{\Phi}(E)$ there exists a constant $C=C(E, \Phi)$ such that

$$
|\{\mathcal{M} f>t\}| \leq \frac{C}{\Phi(t)} \int_{E} \Phi(|f|) d x
$$

for every $t>0$.

Proof We start with standard Chebyshev inequality

$$
|\{\mathcal{M} f>t\}|=\int_{\{\mathcal{M} f>t\} d x \leq \int_{\{\mathcal{M} f>t\}} \Phi(\mathcal{M} f) \Phi(t) d x,}
$$

where we use the fact that Orlicz function $\Phi$ is increasing and $\Phi(\mathcal{M} f)$ is integrable. This latter property, in turn, relying on the integrability of $\Phi(|f|),(5)$ and result the continuity of Hardy's operator in [8]. Assuming that $\Phi$ satisfies (5), (6), [12, Theorem 1] (with applied weight $w \equiv \chi_{\{\mathcal{M} f>t\}}$ note that condition (2) is obviously satisfied) shows that there exists a constant $C>0$ such that

$$
\int_{\{\mathcal{M} f>t\}} \Phi(\mathcal{M} f) \Phi(t) d x \leq \frac{C}{\Phi(t)} \int_{\{\mathcal{M} f>t\} \Phi(|f|) d x}
$$

for every $t>0$.

It is worth to observe that the result holds with the same proof in the vectorial case.

We quote the Fundamental Theorem on Young measures, which will be invoked in the proof of our main results, for more details we refer to [20] (and regarding Young measures generated by gradients to $[13,15])$.

Theorem 3 Let $E \subset \mathbb{R}^{N}$ be a measurable set of finite measure and let $\left(z_{n}\right)$ be a sequence of measurable functions, $z_{n}: E \rightarrow \mathbb{R}^{m}$. Then there exists a subsequence $\left(z_{n_{k}}\right)$ and a weak * measurable map $v: E \rightarrow \mathcal{M}\left(\mathbb{R}^{m}\right)$ such that the following hold:

(i) $\quad v_{x} \geq 0,\left\|v_{x}\right\|_{\mathcal{M}\left(R^{m}\right)=\int_{\mathbb{R}^{m}} d v_{x} \leq 1}$ for a.e. $x \in E$;

(ii) one has (i') $\left\|v_{x}\right\|_{\mathcal{M}=1}$ for a.e. $x \in E$ if and only if

$$
\lim _{R \rightarrow+\infty} \sup _{k}\left|\left\{\left|z_{n_{k}}\right| \geq R\right\}\right|=0
$$


(iii) if $K \subset \mathbb{R}^{m}$ is a compact subset and $\operatorname{dist}\left(z_{n_{k}}, K\right) \rightarrow 0$ in measure, then $\operatorname{supp} v_{x} \subset K$ for a.e. $x \in E$;

(iv) if ( $\left.\mathrm{i}^{\prime}\right)$ holds, then in (iii) one may replace ' $f$ ' with ' 'if and only if';

(v) if $f: E \times \mathbb{R}^{m} \rightarrow \mathbb{R}$ is a normal integrand, bounded from below, then

$$
\liminf _{n \rightarrow+\infty} \int_{E} f\left(x, z_{n_{k}}(x)\right) d x \geq \int_{E} \int_{\mathbb{R}^{m}} f(x, y) d v_{x}(y) d x
$$

(vi) if ( $\left.\mathrm{i}^{\prime}\right)$ holds and if $f: E \times \mathbb{R}^{m} \rightarrow \mathbb{R}$ is Carathéodory and bounded from below, then

$$
\lim _{n \rightarrow+\infty} \int_{E} f\left(x, z_{n_{k}}(x)\right) d x=\int_{E} \int_{\mathbb{R}^{m}} f(x, y) d v_{x}(y) d x
$$

if and only if $\left(f\left(x, z_{n_{k}}(x)\right)\right)$ is equi-integrable. In this case

$$
f\left(x, z_{n_{k}}(x)\right) \rightarrow \int_{\mathbb{R}^{d}} f(x, y) d v_{x}(y) \operatorname{in} L^{1}(E) .
$$

The map $v: E \rightarrow \mathcal{M}\left(\mathbb{R}^{m}\right)$ is called the Young measure generated by $\left(z_{n_{k}}\right)$.

\section{Proofs of Theorems 1 and 2}

This section is devoted to the proof of our main result.

We start by proving a Lemma which generalizes [20, Lemma 8.13] to the Orlicz setting.

Lemma 2 Let $\Phi$ be an Orlicz function satisfying (5) and (6). Let $E \subset \mathbb{R}^{N}$ be a Lebesgue measurable set of finite measure and let $\left(u_{n}\right)$ be a uniformly bounded sequence in $L^{\Phi}\left(E ; \mathbb{R}^{m}\right)$. For any $r>0$ define the standard truncature operators $\tau_{r}: \mathbb{R} \rightarrow \mathbb{R}$ as

$$
\tau_{r}(t):=\left\{\begin{array}{cc}
t & \text { whenever }|t| \leq r \\
r \frac{t}{|t|} & \text { otherwise }
\end{array}\right.
$$

Then there exist a (non-relabelled) subsequence $\left(u_{n}\right)$ and an increasing sequence of positive numbers $r_{n} \rightarrow+\infty$ such that $\tau_{r_{n}} \circ u_{n}$ are $\Phi$-equi-integrable and the measure $\left|\left\{x \in E: \tau_{r_{n}} \circ u_{n} \neq u_{n}\right\}\right| \rightarrow 0$.

Proof By (i) in Theorem 3, we may assume that $\left(u_{n}\right)$ generates the Young measure $v_{x}$ and (iii) therein guarantees that

$$
\int_{E} \int_{\mathbb{R}^{m}} \Phi(|z|) d v_{x}(z) d x<+\infty .
$$

So we have 
$\lim _{r \rightarrow+\infty} \lim _{n \rightarrow \infty} \int_{E} \Phi\left(\left|\tau_{r} \circ u_{n}\right|\right) d x=\lim _{r \rightarrow+\infty} \int_{E} \int_{\mathbb{R}^{m}} \Phi\left(\left|\tau_{r}(z)\right|\right) d v_{x}(z) d x=\int_{E} \int_{\mathbb{R}^{m}} \Phi(|z|) d v_{x}(z) d x$.

where the first equality relies on (vi) of Theorem 3, and the second one on Lebesgue Monotone Convergence theorem. Take $r_{n}$ such that

$$
\lim _{n \rightarrow+\infty} \int_{E} \Phi\left(\left|\tau_{r_{n}} \circ u_{n}\right|\right) d x=\int_{E} \int_{\mathbb{R}^{m}} \Phi(|z|) d v_{x}(z) d x .
$$

As $r_{n} \rightarrow+\infty$ and $\left(u_{n}\right)$ is bounded, one has

$$
\left|\left\{x \in E: \tau_{r_{n}} \circ u_{n} \neq u_{n}\right\}\right| \rightarrow 0 .
$$

Thus, we can conclude that $\left(\tau_{r_{n}} \circ u_{n}\right)$ generates the same Young measure as $\left(u_{n}\right)$ (see [20, Corollary 8.7]).

Finally (vi) in Theorem 3 ensures $\Phi$-equi-integrability.

Now we prove a Decomposition Lemma for gradients and then we extend this result to scaled ones.

Theorem 4 Let $E \subset \mathbb{R}^{N}$ be a bounded open set with Lipschitz boundary. Let $\Phi$ be an Orlicz function satisfying (5) and (6), and let $\left(u_{n}\right) \subset W^{1, \Phi}\left(E ; \mathbb{R}^{d}\right)$ be a sequence of functions converging to $u_{0}$ weakly in $W^{1, \Phi}\left(E ; \mathbb{R}^{d}\right)$. Then there exists a subsequence $\left(u_{n_{k}}\right)$ and a sequence $\left(v_{k}\right) \subset W^{1, \infty}\left(\mathbb{R}^{N} ; \mathbb{R}^{d}\right)$ such that $\left(v_{k}\right)$ converges to $u_{0}$ weakly in $W^{1, \Phi}\left(E ; \mathbb{R}^{d}\right)$, and

$$
\mid\left\{x \in E: v_{k}(x) \neq u_{k}(x) \text { or } \nabla u_{k}(x) \neq \nabla v_{k}(x)\right\} \mid \rightarrow 0 \text { as } k \rightarrow+\infty
$$

and $\left(\Phi\left(\left|\nabla v_{k}\right|\right)\right)$ is equi-integrable.

Proof Since

$$
\sup _{n}\left\|u_{n}\right\|_{W^{1, \Phi}\left(E ; \mathbb{R}^{d}\right)} \leq C
$$

and by (5),

$$
\sup _{n}\left\{\int_{E}\left(\Phi\left(\left|u_{n}\right|\right)+\Phi\left(\left|\nabla u_{n}\right|\right)\right) d x\right\} \leq C,
$$

it follows that from the continuity of the maximal operator [8, Theorem 2.1], and the passage to an equivalent norm, that

$$
\sup _{n}\left\{\int_{\mathbb{R}^{N}} \Phi\left(\mathcal{M}\left(\left|u_{n}\right|+\left|\nabla u_{n}\right|\right) \chi_{E}\right) d x\right\} \leq C,
$$

where $\mathcal{M}\left(\left(\left|u_{n}\right|+\left|\nabla u_{n}\right|\right) \chi_{E}\right)$ is the maximal function of $\left(\left|u_{n}\right|+\left|\nabla u_{n}\right|\right) \chi_{E}$. By Lemma 2, there exists an increasing sequence $t_{n} \rightarrow+\infty$ such that $\left(\Phi\left(\mid \tau_{t_{n}} \circ\right.\right.$ $\left.\left.\left(\mathcal{M}\left(\left(\left|u_{n}\right|+\left|\nabla u_{n}\right|\right) \chi_{E}\right)\right) \mid\right)\right)$ is equi-integrable, where $\tau_{t_{n}}$ is as in (10).

Define 


$$
A_{n}:=\left\{x \in E:\left|\mathcal{M}\left(\left(\left|u_{n}\right|+\left|\nabla u_{n}\right|\right) \chi_{E}\right)\right|>t_{n}\right\} .
$$

By [20, Theorem 4.32], there exists $\left(v_{n}\right) \subset W^{1, \infty}\left(\mathbb{R}^{N} ; \mathbb{R}^{m}\right)$ such that

$$
\left\|v_{n}\right\|_{W^{1, \infty}} \leq C t_{n},
$$

where $C$ depends on $E$ and $N$, and such that $v_{n}=u_{n} \mathcal{L}^{N}$ a.e. on $E \backslash A_{n}$ and by (9)

$$
\left|A_{n}\right| \leq \frac{C}{\Phi(t)} \int_{\mathbb{R}^{N}} \Phi\left(\left|u_{n}\right|+\left|\nabla u_{n}\right|\right) d x .
$$

In order to show that $\left(\Phi\left(\left|\nabla v_{n}\right|\right)\right)$ is equi-integrable we observe that for $\mathcal{L}^{N}$ a.e. $x$ in $E \backslash A_{n}$

$$
\left|\nabla v_{n}\right|=\left|\nabla u_{n}\right| \leq \mathcal{M}\left(\left(\left|u_{n}\right|+\left|\nabla u_{n}\right|\right) \chi_{E}\right)=\left|\tau_{t_{n}} \circ \mathcal{M}\left(\left(\left|u_{n}\right|+\left|\nabla u_{n}\right|\right) \chi_{E}\right)\right|
$$

while if $x \in A_{n}$ then

$$
\left|\nabla v_{n}\right| \leq C t_{n} \leq C\left|\tau_{t_{n}} \circ \mathcal{M}\left(\left(\left|u_{n}\right|+\left|\nabla u_{n}\right|\right) \chi_{E}\right)\right| .
$$

It remains to prove the weak convergence of $\left(v_{n}\right)$ to $u_{0}$ in $W^{1, \Phi}\left(E ; \mathbb{R}^{d}\right)$. To this end, first we observe that (11) and (9) ensure

$$
\begin{aligned}
\int_{E} \Phi\left(\left|v_{n}\right|+\left|\nabla v_{n}\right|\right) d x & =\int_{E \backslash A_{n}} \Phi\left(\left|u_{n}\right|+\left|\nabla u_{n}\right|\right) d x+\int_{A_{n}} \Phi\left(\left|v_{n}\right|+\left|\nabla v_{n}\right|\right) d x \\
& \leq \int_{E \backslash A_{n}} \Phi\left(\left|u_{n}\right|+\left|\nabla u_{n}\right|\right) d x+C \Phi\left(t_{n}\right)\left|A_{n}\right| \\
& \leq C \int_{E} \Phi\left(\left|u_{n}\right|+\left|\nabla u_{n}\right|\right) d x .
\end{aligned}
$$

Next the reflexivity of $W^{1, \Phi}\left(E ; \mathbb{R}^{d}\right)$ under (5), (6) (see Lemma 1) and the BanachAlaoglu-Bourbaki theorem ensure that $v_{n} \rightarrow v_{0}$ in $W^{1, \Phi}\left(E ; \mathbb{R}^{d}\right)$. Thus, since $\mid\{x \in$ $E: v_{n} \neq u_{n}$ or $\left.\nabla u_{n} \neq v_{n}\right\} \mid \rightarrow 0$ as $n \rightarrow+\infty$ we can conclude, via the compact imbedding (see (8)) that $v_{0}=u_{0} \mathcal{L}^{N}$-a.e. in $E$.

Proof of Theorem 1 The proof of the claims (i) and (iii) follows line by line as in [4, Theorem 3.1]. Namely, we define $\hat{u}_{n}:=u_{n}\left(x_{1}, x_{2}, \frac{x_{3}}{\varepsilon_{j}}-1\right.$ ) (so it is a shifted and scaled version of $u_{n}$, and it is defined on $\left.\omega \times\left(0,2 \varepsilon_{n}\right)\right)$ and observe that

$$
\sup _{j} \varepsilon_{j}^{-1} \int_{\omega \times\left(0,2 \varepsilon_{n}\right)} \Phi\left(\left|\nabla \hat{u}_{n}\right|\right) d x=C, \quad \text { where Cisexactlylikein(1.2). }
$$

We now extend $\hat{u}_{n}$ by reflection to $\omega \times\left(-2 \varepsilon_{n}, 2 \varepsilon_{n}\right)$ and then produce its periodic extension to $\omega \times(-1,1)$.

For such constructed sequence one can obtain the uniform bound of the norm in $W^{1, \Phi}(\omega \times(-1,1))$ as in $[4$, formula (3.6)]. Thus we apply Theorem 4 and obtain a sequence $\left(\hat{v}_{n}\right)$ with $\left(\nabla \hat{v}_{n}\right) \Phi$-equi-integrable. The use of de la Vallée Poussin Criterion (see [20, Theorem 2.29]) and an ingenious computation (see [4, formula 
(3.7)]) gives us the sequence $\left(\bar{v}_{n}\right)$ satisfying claim (i) and (iii).

Up to an extraction of a subsequence one may immediately deduce claim (ii).

To get (iv) we argue as in [3, Corollary 1.2]. We define sets

$$
\omega_{j}:=\{x \in \omega: \operatorname{dist}(x, \partial \omega)<1 / j\}
$$

and cut-off functions $\theta_{j} \in C_{0}^{\infty}(\omega,[0,1])$, equal to 1 on $\omega \backslash \omega_{j}$, vanishing in a neighbourhood of $\partial \omega$, and such that $\left|\nabla \theta_{j}\right|<C j$ for some constant $C$. We set then $v_{n, j}:=u_{0}+\theta_{j} \bar{v}_{n}$. Via compact imbedding (see (8)) and diagonal argument we may find a sequence $n(j)$ such that $n(j) \rightarrow+\infty$ as $j \rightarrow+\infty$ and

$$
\left\|v_{n(j), j}-u_{0}\right\|_{L^{\Phi}\left(\Omega ; \mathbb{R}^{3}\right)} \rightarrow 0 \quad \text { and } \quad\left\|v_{n(j), j}\right\|_{L^{\Phi}\left(\Omega ; \mathbb{R}^{3}\right)}<\frac{1}{j^{2}} .
$$

To obtain (iv), it suffices to define $v_{j}:=v_{n(j), j}$. It remains to deduce (i)-(iii) for this latter sequence. To prove (iii) we just observe that

$$
\begin{aligned}
& \left|\left\{x \in \Omega: u_{j} \neq v_{j} \operatorname{or} \nabla u_{j} \neq \nabla v_{j}\right\}\right| \\
& \quad \leq \mid\left\{x \in \Omega: u_{j} \neq \bar{v}_{j} \text { or } \nabla u_{j} \neq \nabla \bar{v}_{j}\right\}|+|\left\{x \in \Omega: \bar{v}_{j} \neq v_{j} \text { or } \nabla u_{j} \neq \nabla \bar{v}_{j}\right\} \mid,
\end{aligned}
$$

and the claim follows from the control of the latter two sets. For (i), it suffices to exploit the definition of $u_{j}$ and the $\Phi$-equi-integrability of $\bar{v}_{j}$, (see also [3, formula (4.8)]). Up to the extraction of the subsequence we may know deduce (ii).

Proof of Theorem 2 It can be deduced from [3, Corollary 1.2]. We sketch the main points for the readers' convenience. First let us observe that from density of smooth functions and properties of quasiconvex envelope and definition of $\bar{W}$ it can be easily proven that

$$
\inf _{\varepsilon,\left.u\right|_{\partial \omega \times(-1,1)} \equiv u_{0}} \frac{1}{|\Omega|} \int_{\Omega} W\left(\nabla_{\alpha} u, \frac{1}{\varepsilon} \nabla_{3} u\right) d x=Q \bar{W}\left(\xi_{0}\right) .
$$

Now let us assume that $\omega$ is a square $(-c / 2, c / 2)^{2}$. Let $\left(w_{n}, L_{n}\right)$ be the infimizing sequence of the left-hand side in (13). We may thus assume that, up to a reflection and then a periodic extension, functions $\left(w_{n}-u_{0}\right)$ are already defined on $\mathbb{R}^{2} \times$ $(-1,1)$. We define $w_{n, j}(x):=\varepsilon_{j} L_{n}\left(w_{n}-u_{0}\right)\left(\left(\varepsilon_{j} L_{n}\right)^{-1} x_{\alpha}, x_{3}\right)$ and observe that $w_{n, j} \rightarrow$ 0 and

$$
\lim _{n \rightarrow \infty} \lim _{j \rightarrow \infty} \frac{1}{|\Omega|} \int_{\Omega} W\left(\nabla_{\alpha} u_{0}+\nabla_{\alpha} w_{n, j}, \frac{1}{\varepsilon_{j}} \nabla_{3} w_{n, j}\right)=Q \bar{W}\left(\xi_{0}\right) .
$$

By a diagonal procedure and (8) we may choose $j(n)$ such that (denoting $w_{n, j(n)}$ as $\tilde{w}_{n}$ and $\varepsilon_{j(n)}$ as $\left.\tilde{\varepsilon}_{n}\right), \lim \tilde{w}_{n}=0$ in $L^{\Phi}(\Omega)$, and

$$
\lim _{n \rightarrow \infty} \frac{1}{|\Omega|} \int_{\Omega} W\left(\nabla_{\alpha} \tilde{w}_{n}, \frac{1}{\tilde{\varepsilon}_{n}} \nabla_{3} \tilde{w}_{n}\right) d x=Q \bar{W}\left(\xi_{0}\right) .
$$

The latter equality together with (2) gives us bound on the norm of $\tilde{w}_{n}$ in $W^{1, \Phi}\left(\Omega ; \mathbb{R}^{3}\right)$. Up to an extraction of the subsequence (not relabelled) we may still 
assume that $\tilde{w}_{n} \rightarrow 0$.

Applying Theorem 1 we obtain a sequence $\left(v_{n}\right)$ satisfying (ii)-(iv). (i) follows from triangle inequality, $\Phi$-equi-integrability of $\left(v_{n}\right)$, point (iii) and the fact that $\left|\omega_{j}\right| \rightarrow 0$ (see (12)).

To generalize the result to $\omega$ with Lipschitz boundary we refer to the second step of the proof of [3, Corollary 1.2].

Acknowledgements Research supported by WCMCS, http://www.wcmcs.edu.pl/ and by INdAMGNAMPA through the project 'Professori Visitatori 2016'.

Open Access This article is distributed under the terms of the Creative Commons Attribution 4.0 International License (http://creativecommons.org/licenses/by/4.0/), which permits unrestricted use, distribution, and reproduction in any medium, provided you give appropriate credit to the original author(s) and the source, provide a link to the Creative Commons license, and indicate if changes were made.

\section{References}

1. Acerbi, E., Fusco, N.: Semicontinuity problems in the calculus of variations. Arch. Ration. Mech. Anal. 86(2), 125-145 (1984)

2. Adams, R.A.: On the Orlicz-Sobolev imbedding theorem. J. Funct. Anal. 24, 241-257 (1977)

3. Bocea, M., Fonseca, I.: Equi-integrability results for 3d-2d dimension reduction problems. ESAIM: COCV 7, 443-470 (2002)

4. Braides, A., Zeppieri, C.I.: A note on equi-integrability in dimension reduction problems. Calc. Var. 29, 231-238 (2007)

5. Carita, G., Zappale, E.: 3D-2D dimensional reduction for a nonlinear optimal design problem with perimeter penalization. CRAS Math. 350, 1011-1016 (2012)

6. Carita, G., Zappale, E.: Relaxation for an optimal design problem with linear growth and perimeter penalization. Proc. R. Soc. Edinb. Sect. A 145, 223-268 (2015)

7. Fonseca, I., Müller, S., Pedregal, P.: Analysis of concentration and oscillation effects generated by gradients. SIAM J. Math. Anal. 29(3), 736-756 (1998) (electronic)

8. Gallardo, Diego: Orlicz spaces for which the Hardy-Littlewood maximal operator is bounded. Publicacions Matemtiques 32, 261-266 (1988)

9. García-Huidobro, M., Le, V.K., Manásevich, R., Schmitt, K.: On principal eigenvalues for quasilinear elliptic differential operators: an Orlicz-Sobolev space setting. NoDEA Nonlinear Differ. Equ. Appl. 6(2), 207-225 (1999)

10. Gossez, J.-P.: Some approximation properties in Orlicz-Sobolev spaces. Studia Mathematica 74(1), 17-24 (1982). (eng)

11. Kałamajska, A., Krbec, M.: Traces of Orlicz-Sobolev functions under general growth restrictions. Mathematische Nachrichten 286(7), 730-742 (2013)

12. Kerman, R., Torchinsky, A.: Integral inequalities with weights for the Hardy maximal function. Stud. Math. 71(3), 277-284 (1982)

13. Kinderlehrer, D., Pedregal, P.: Characterizations of Young measures generated by gradients. Arch. Ration. Mech. Anal. 115(4), 329-365 (1991)

14. Kinderlehrer, D., Pedregal, P.: Weak convergence of integrands and the Young measure representation. SIAM J. Math. Anal. 23(1), 1-19 (1992)

15. Kinderlehrer, D., Pedregal, P.: Gradient Young measures generated by sequences in Sobolev spaces. J. Geometr. Anal. 4(1), 59-90 (1994)

16. Kozarzewski, P., Zappale, E.: A note on optimal design for thin structures in the Orlicz-Sobolev setting. In: Proceedings for the IMSE2016 Conference (to appear)

17. Krasnosel'skiǔ, M.A., Rutickiǐ, I.B.: Convex functions and Orlicz spaces. In: Volume 8 of Russian Monographs and Texts on Advanced Mathematics and Physics, 249 p, P.Noordhoff (1961) 
18. Laskowski, W., Nguyêñ, H.: Effective energy integral functionals for thin films in the OrliczSobolev space setting. Demonstr. Math. XLVI(3), 7-31 (2013)

19. Laskowski, W., Nguyêñ, H.T.: Effective energy integral functionals for thin films with bending moment in the Orlicz-Sobolev space setting. Banach Cent. Publ. 102, 143-167 (2014)

20. Leoni, G., Fonseca, I.: Modern methods in the calculus of variations: $L^{p}$ spaces. Springer monographs in mathematics. Springer, New York (2007)

21. Tachago, J.F., Nnang, H.: Two-scale convergence of integral functionals with convex, periodic and nonstandard growth integrands. Acta Appl. Math. 121, 175-196 (2012) 